\section{Commentary: Reefing the sails and the direction of the wind}

\author{
Tomasz A. Timek, MD, PhD
}

The transcatheter valve technologies that have revolutionized our approach to aortic valve pathology ${ }^{1}$ are now beginning to be harnessed to address mitral regurgitation ${ }^{2}$ in prohibitive-risk patients. The mitral valve represents a greater technical challenge due to its complex structure, ill-defined annulus, and potential for left ventricular outflow tract (LVOT) obstruction due to the displacement of the anterior mitral leaflet (AML). Identifying patients with favorable anatomical features continues to be a challenge ${ }^{3}$ but is of critical importance, as LVOT obstruction during transcatheter mitral valve replacement (MVR) is associated with up to $34 \%$ procedural mortality. ${ }^{4}$ Percutaneous techniques of anterior leaflet laceration ${ }^{5}$ and balloon translocation ${ }^{6}$ have been developed to mitigate this risk. However, even with successful percutaneous valve deployment, it is unknown how altered geometry and motion of the AML may affect ventricular flow, function, and energetics.

In the current issue of the Journal, Brunel and colleagues ${ }^{7}$ from the University of Sydney present an innovative ovine study investigating the hemodynamic effects of reversibly reefing the AML after implantation of a mechanical mitral valve prosthesis. These investigators found that incorporating the anterior leaflet into the anterior annulus was associated with a reduction in load-dependent and -independent markers of myocardial performance and deleterious effects on left ventricular geometry. The authors

\footnotetext{
From the Division of Cardiothoracic Surgery, Spectrum Health/Michigan State University College of Human Medicine, Grand Rapids, Mich.

Disclosures: The author reported no conflicts of interest.

The Journal policy requires editors and reviewers to disclose conflicts of interest and to decline handling or reviewing manuscripts for which they may have a conflict of interest. The editors and reviewers of this article have no conflicts of interest.

Received for publication April 22, 2021; revisions received April 22, 2021; accepted for publication April 26, 2021; available ahead of print June 8, 2021.

Address for reprints: Tomasz A. Timek, MD, PhD, Division of Cardiothoracic Surgery, Spectrum Health/Michigan State University College of Human Medicine, 100 Michigan Ave NE, Grand Rapids, MI 49503 (E-mail: tomasz.timek@ spectrumhealth.org).

JTCVS Open 2021;7:121-2

2666-2736

Copyright (C) 2021 The Authors. Published by Elsevier Inc. on behalf of The American Association for Thoracic Surgery. This is an open access article under the CC BY-NCND license (http://creativecommons.org/licenses/by-nc-nd/4.0/).

https://doi.org/10.1016/j.xjon.2021.04.014
}

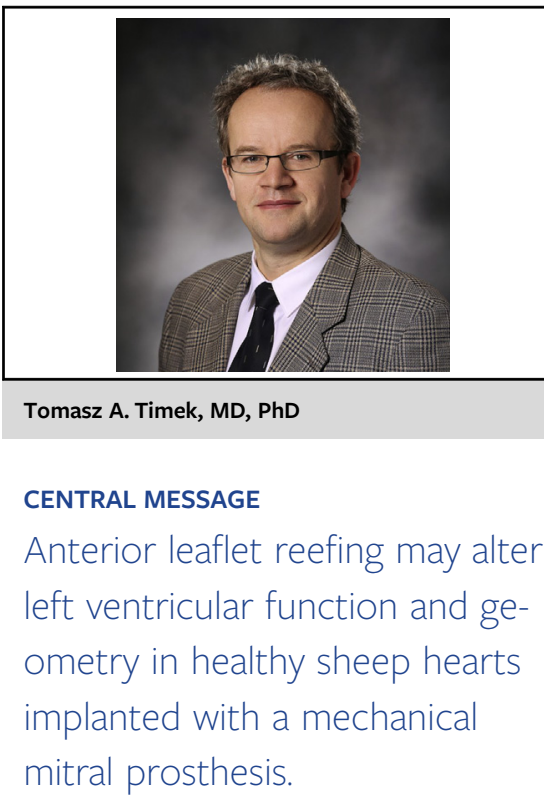

concluded that percutaneous mitral implants, which similarly immobilize the anterior leaflet, may impair left ventricular myocardial function.

The described experiment represents an elegant approach of interrogating in isolation a component of the mitral valve apparatus in the setting of an implanted mitral prosthesis, yet in many ways it departs from clinical reality. Currently there is no surgical technique that duplicates this approach clinically, but the authors are attempting to simultaneously extrapolate the results of the study to both chordal-sparing MVR and transcatheter MVR, which may not be appropriate. Anterior leaflet-sparing MVR reefs the AML to the annulus by incorporating the leaflet in the interrupted sutures that anchor the mitral valve. This is distinctively different from reefing the leaflet after the mitral valve prosthesis is in place. In the current experimental protocol, the AML is being reefed "behind" the anterior annulus and into the LVOT. It is feasible that subclinical LVOT obstruction may be a significant contributor to the altered hemodynamics that the investigators report during leaflet reefing. Clinically, even with a low-profile mechanical mitral prosthesis, LVOT obstruction has been reported with preservation of AML. ${ }^{8}$ Neither thorough echocardiographic assessment of LVOT nor direct pressure measurements between the LV and aorta were taken during leaflet reefing to exclude this possibility. Alterations of intracardiac flow patterns may also contribute to the reported findings. Native geometry and motion of the AML facilitate diastolic chamber filling and left ventricular ejection whereas mitral valve repair and replacement incrementally disturb normal flow 
patterns. ${ }^{9}$ The choice of a mechanical prosthesis further obfuscates the message, as left ventricular filling patterns can be expected to be further perturbed by a bileaflet mechanical prosthesis. Lastly, direct suture traction to reef the leaflet may have altered geometry of the aortic root and induced aortic insufficiency that may further contribute to hemodynamic changes and alterations of left ventricular geometry. The presented experimental study is thought-provoking and adds to our basic understanding of mitral valve physiology, but any clinical extrapolation should be viewed through a prism of multiple important limitations.

\section{References}

1. Makkar RR, Thourani VH, Mack MJ, Kodali SK, Kapadia S, Webb JG, et al. Fiveyear outcomes of transcatheter or surgical aortic-valve replacement. $N$ Engl J Med. 2020;382:799-809.

2. Hensey M, Brown RA, Lal S, Sathananthan J, Ye J, Cheung A, et al. Transcatheter mitral valve replacement: an update on current techniques, technologies, and future directions. JACC Cardiovasc Interv. 2021;14:489-500.
3. Ludwig S, Koell B, Sedighian R, Weimann J, Schofer N, Schäfer A, et al. Characteristics and outcomes of patients ineligible for transcatheter mitral valve replacement (TMVR): implications for future device innovation. J Invasive Cardiol. 2021;33:E294-301.

4. Yoon SH, Bleiziffer S, Latib A, Eschenbach L, Ancona M, Vincent F, et al. Predictors of left ventricular outflow tract obstruction after transcatheter mitral valve replacement. JACC Cardiovasc Interv. 2019;12:182-93.

5. Khan JM, Babaliaros VC, Greenbaum AB, Foerst JR, Yazdani S, McCabe JM, et al. Anterior leaflet laceration to prevent ventricular outflow tract obstruction during transcatheter mitral valve replacement. J Am Coll Cardiol. 2019;73: 2521-34.

6. Helmy T, Hui DS, Smart S, Lim MJ, Lee R. Balloon assisted translocation of the mitral anterior leaflet to prevent left ventricular outflow obstruction (BATMAN): a novel technique for patients undergoing transcatheter mitral valve replacement. Catheter Cardiovasc Interv. 2020;95:840-8.

7. Brunel L, Williams ZA, Yata M, Robinson BM, Wise IK, Paterson HS, et al. Incorporating the anterior mitral leaflet to the annulus impairs left ventricular function in an ovine model. J Thorac Cardiovasc Surg Open. 2021;7:111-20.

8. Melero JM, Rodriguez I, Such M, Porras C, Olalla D. Left ventricular outflow tract obstruction with mitral mechanical prosthesis. Ann Thorac Surg. 1999; 68:255-7.

9. Yoshida S, Miyagawa S, Fukushima S, Yoshikawa Y, Hata H, Saito S, et al. Cardiac function and type of mitral valve surgery affect postoperative blood flow pattern in the left ventricle. Circ J. 2018;83:130-8. 